\title{
Les parents québécois et l'État canadien au début du programme des allocations familiales : 1944-1955
}

\section{Dominique Jean}

Volume 40, numéro 1, été 1986

URI : https://id.erudit.org/iderudit/304425ar

DOI : https://doi.org/10.7202/304425ar

Aller au sommaire du numéro

\section{Éditeur(s)}

Institut d'histoire de l'Amérique française

\section{ISSN}

0035-2357 (imprimé)

1492-1383 (numérique)

Découvrir la revue

Citer cet article

Jean, D. (1986). Les parents québécois et l’État canadien au début du programme des allocations familiales : 1944-1955. Revue d'histoire de l'Amérique française, 40(1), 73-95. https://doi.org/10.7202/304425ar
Résumé de l'article

Quand le gouvernement King instaure un programme d'allocations familiales en 1945, il compte s'attirer les faveurs des électorats ouvrier et québécois, tous deux mécontents de sa façon de diriger le pays pendant la guerre. L'examen des effets du programme au Québec dans la première décennie montre que, dans les deux cas, le premier programme de sécurité sociale au Canada a été apprécié. Les règlements des allocations prévoient un certain contrôle étatique de l'économie et de la structure familiale. Mais le versement régulier d'une somme d'argent à tous les parents est assez apprécié pour que les rares intrusions dans les foyers québécois n'affectent pas les 96 pour cent de l'opinion publique qui approuve le programme. Les aspirations des ménages pauvres sont rencontrées par [les aspects de la loi qui promeuvent] la famille naturelle comme meilleur environnement pour le développement de l'enfant, de même que par le niveau de confiance élevé accordé aux parents par le gouvernement [quant à la dépense de l'allocation] quand on compare les modalités du programme aux méthodes discrétionnaires des organisations charitables. Des séquelles de cette approche subjective sont cependant reprises pour les allocations aux immigrants et aux autochtones. Enfin, les familles québécoises auraient pu, comme une importante section de leur élite, s'élever contre le versement du chèque à la mère, la menace de l'autonomie familiale et le taux décroissant après le cinquième enfant. En pratique, au contraire, la majorité des mères administrent le budget familial, et les familles nombreuses sont celles dont le revenu subit l'augmentation relative la plus importante. Ainsi, les dénonciations autonomistes de Duplessis et le boycott organisé par le Bloc populaire ne recevront pas l'appui escompté.
Tous droits réservés @ Institut d'histoire de l'Amérique française, 1986

Ce document est protégé par la loi sur le droit d'auteur. L'utilisation des services d'Érudit (y compris la reproduction) est assujettie à sa politique d'utilisation que vous pouvez consulter en ligne.

https://apropos.erudit.org/fr/usagers/politique-dutilisation/ 


\title{
LES PARENTS QUÉBÉCOIS ET L'ÉTAT CANADIEN AU DÉBUT DU PROGRAMME DES ALLOCATIONS FAMILIALES: 1944-1955
}

\author{
DOMINIQUE JEAN ${ }^{1}$ \\ Étudiante au doctorat \\ Département d' histoire \\ Université de Montréal
}

\section{RÉSUMÉ}

Quand le gouvernement King instaure un programme d'allocations familiales en 1945, il compte s'attirer les faveurs des électorats ouvrier et québécois, tous deux mécontents de sa façon de diriger le pays pendant la guerre. L'examen des effets du programme au Québec dans la première décennie montre que, dans les deux cas, le premier programme de sécurité sociale au Canada a été apprécié. Les réglements des allocations prévoient un certain contrôle étatique de l'économie et de la structure familiale. Mais le versement régulier d'une somme d'argent à tous les parents est assez apprécié pour que les rares intrusions dans les foyers québécois n'affectent pas les 96 pour cent de l'opinion publique qui approuve le programme. Les aspirations des ménages pauvres sont rencontrées par [les aspects de la loi qui promeuvent] la famille naturelle comme meilleur environnement pour le développement de l'enfant, de même que par le niveau de confiance élevé accordé aux parents par le gouvernement [quant à la dépense de l'allocation] quand on compare les modalités du programme aux méthodes discrétionnaires des organisations charitables. Des séquelles de cette approche subjective sont cependant reprises pour les allocations aux immigrants et aux autochtones. Enfin, les familles québécoises auraient pu, comme une importante section de leur élite, s'élever contre le versement du chèque à la mère, la menace de l'autonomie familiale et le taux décroissant après le cinquième enfant. En pratique, au contraire, la majorité des mères administrent le budget familial, et les familles nombreuses sont celles dont le revenu subit l'augmentation relative la plus impor-

1 Je tiens à remercier René Durocher, Bettina Bradbury et Claude Couture, de l'Université de Montréal, Benneth McCardle des Archives publiques du Canada, John Taylor de la bibliothèque du ministère de la Santé nationale et du Bien-être social et Philippe Jean pour leur aide et leurs précieux conseils. 
tante. Ainsi, les dénonciations autonomistes de Duplessis et le boycott organisé par le Bloc populaire ne recevront pas l'appui escompté.

\begin{abstract}
With the 1945 family allowances programme, the King government hoped to win back segments of the electorate, especially Quebecers and workers, who were disappointed with its wartime policies. This study of the effects of the programme in Quebec from 1945 to 1955 shows that, in both cases, the first universal Canadian social security programme was appreciated. True, this measure contained provisions meant to control the family economy and structure. But the regular family allowance payment to mothers was so popular that public approval of the programme reached 96 per cent. Poor households' expectations were met by the promotion of the family, as opposed to institutions, as the best means of raising children. Another favorable aspect was the confidence given to parents to spend the allowance, especially when the programme is compared to former private charity measures. More control, however, was exercised in the cases of immigrants and natives. Finally, Quebec families could have protested family allowances and the decreasing rate after the fifth child, as did a substantial section of their elite, but the financial needs of most mothers, who were responsible for administering the family budget, prevented them from following the Bloc populaire's boycott and Duplessis' efforts to protect provincial autonomy.
\end{abstract}

En recevant leur premier chèque d'allocations familiales en juillet 1945 , plus de 350000 mères québécoises ouvrent la porte de leur foyer à l'État, la plupart pour la première fois. L'application du premier programme universel de sécurité sociale au Canada inaugure une nouvelle ère de relations entre les familles et le gouvernement fédéral. L'étude de ces interactions, pour la décennie qui suit, révèle à la fois les normes économiques et culturelles attachées à l'enfance par les élites gouvernementales fédérales de l'après-guerre, et les stratégies internes des familles pour s'ajuster aux changements extérieurs de ce milieu du $20 \mathrm{e}$ siècle.

Les années 1945-1955 sont d'autant plus importantes qu'on y définit, pour les enfants, un statut qui s'inscrit encore dans les lois et les politiques sociales. Les décennies subséquentes en ont expérimenté la logique, dénoncé les faiblesses et les contradictions. Ainsi, les récents débats sur l'universalité des allocations familiales, leur indexation et 
leur abolition au profit du seul crédit-impôt pour enfants, annoncent le besoin d'un nouveau consensus ${ }^{2}$.

La Loi des allocations familiales et les règlements afférents ${ }^{3}$ imposent des conditions au versement de l'allocation qui touchent le statut économique et social des enfants. Ils demandent que la prestation soit dépensée pour l'enfant, exigent au préalable un minimum d'entretien par les parents, nécessitent la fréquentation scolaire jusqu'à 16 ans, interdisent le travail juvénile à plein temps et désignent très précisément qui peut recevoir les prestations pour les enfants orphelins ou abandonnés.

Cette tranche de l'étude de l'implantation du régime des allocations, dont l'ensemble constitue une thèse de doctorat, examine les deux premières conditions énoncées plus haut pour tenter d'établir dans quelle mesure le rôle de l'État entre en conflit avec le rôle des parents. Notre cadre d'interprétation accorde une importance égale aux efforts de contrôle social et aux réponses humaines à ces efforts. Cette perspective, qui suppose une relation dynamique entre l'État et les familles, rallie de plus en plus d'historiens de la famille, de l'éducation et de l'enfance, depuis la fin des années 1970 . Elle présente les familles non uniquement comme objets mais aussi comme agents du changement social. Dans le cas du programme des allocations familiales, cette approche permet de découvrir une remarquable différence entre d'une part l'ouverture des familles et d'autre part l'opposition de la majorité de l'élite clérico-nationaliste de la province.

C'est à une structure administrative décentralisée, et à l'efficacité dont les fonctionnaires fédéraux de l'heure font leur credo, que nous devons les rapports du Bureau régional de Québec qui forment le principal corpus documentaire de cette étude: d'imposants bureaux régionaux des allocations familiales, mis sur pied en quelques mois dans toutes les capitales provinciales, doivent appliquer les restrictions en plus d'assurer la distribution des chèques. Huit enquêtes sur les effets des allocations complètent la documentation. Elles ont été dirigées par des sociologues, des travailleurs sociaux ou des fonctionnaires, entre 1947 et 1951. Leur rigueur méthodologique varie, de même que leur méthode d'échantillonnage. Mais, malgré tout, cet ensemble d'informations hétérogènes contient de nombreux indices significatifs du comportement et de l'opinion de centaines de familles ${ }^{4}$.

₹ Cette notion de consensus est entendue au sens où Brigitte Kitchen la présente dans «The Family and Social Policy», in Maureen Baker, ed., The Family: Changing Trends in Canada (McGraw-Hill Ryerson, 1984), 181-182.

3 «Loi de 1944 sur les allocations familiales», Statuts du Canada, 1944-45, c. 40, 8 George VI; «Règlements sur les allocations familiales», Gazette du Canada, 79,34 (25 août 1945).

4 Quatre études excluent délibéremment les familles où le père ou la mère sont absents. Comment peut-on mettre le doigt sur des modèles familiaux non-dominants quand on choisit de ne pas tenir compte des situations conflictuelles? Voir la critique d'Huguette Dagenais, «Images 


\section{1 - Une loi sociale aux visées d'abord économiques}

Les législateurs sont certes préoccupés par les enfants pour le bienêtre et l'égalité des chances desquels la mesure est annoncée: ils leur dédient le premier programme de sécurité sociale. A une époque où les prestations publiques ont encore un caractère stigmatisant et ne sont souvent pas perçues comme un droit, «il n'est pas surprenant que les changements dans le domaine du bien-être social soient dirigés vers les secteurs qui bénéficient déjà de sympathie: vers ceux qui sont dans le besoin sans pouvoir en porter la responsabilité, comme les veuves et les enfants ${ }^{5}$. Les allocations ne s'adressent pas directement aux enfants cependant, mais aux familles. C'est en effet à travers elles, et en les aidant à jouer leur rôle, que le gouvernement entend aider les enfants.

Pourtant, le bien-être des familles canadiennes et de leurs enfants représente une préoccupation secondaire du gouvernement fédéral de 1944. Les rapports et la correspondance qui nous sont parvenus des politiciens et des hauts fonctionnaires canadiens responsables de la planification du passage à l'économie de paix indiquent en effet que la Loi des allocations familiales, votée le 15 août 1944, représente avant tout un sauvetage de la politique de stabilisation des prix et des salaires, instaurée en 1941 pour enrayer l'inflation de guerre, et fortement ébranlée par la contestation syndicale dès 1942. Les historiens Breul et Kitchen ont montré comment la proposition des allocations énoncée, en 1944, dans le rapport de la Commission nationale du travail en temps de guerre chargée d'enquêter sur les relations de travail au Canada, à la suite de la grève des travailleurs de l'acier, a eu plus d'influence sur le gouvernement King que le Rapport Marsh de 1943 sur la sécurité sociale de la reconstruction. La Commission nationale du travail en temps de guerre suggérait en fait deux alternatives: la déréglementation des salaires inférieurs à 50 cents de l'heure, comme le demandaient les syndicats (là où les salaires avaient été retirés des objets des négociations collectives pour la durée de la guerre), ou les allocations ${ }^{6}$. En 1943, le rapport Marsh avait pour sa part proposé des allocations fami-

\footnotetext{
de femmes dans les monographies sur la famille et la société canadiennes-françaises et québécoises», communication présentée au colloque de l'ICRAF, 10 novembre 1984. Il s'agit de R. Blishen, J. Cawley et J. E. Pearson, Family Allowances in Montreal; a Study of their Uses and Meaning in a Selected Group of Wage Earning Families, Thèse de M. A. (Travail social), Université McGill, 1948; Derek Griffin, Family Budgets of Wage-Earners in Four Maritime Communities. 1947 (Halifax, The Institute of Public Affairs, Dalhousie University, 1952); Thérèse Légaré, Conditions économiques et sociales des familles de Gaspé-Nord (Québec, Faculté des sciences sociales, Université Laval, mai 1947), document no 1 du Centre de Recherches (Service social); Thérèse Roy, Influence économique et sociale des allocations familiales, thèse de M. A. (Relations industrielles), Université de Montréal, 1948.

5 Elwood Jones, «Dependency and Social Welfare», Journal of Canadian Studies/Revue d'études canadiennes, 14,1 (Spring 1979): 2. Notre traduction.

6 Frank Breul, «The Genesis of Family Allowances in Canada», The Social Service Review, 27,3 (Sept. 1953): 269-280; Brigitte Kitchen, «Wartime Social Reform: the Introduction of Family Allowances», Revue canadienne d'éducation en service social, 7,1 (1981): 29-54.
} 
liales comme moyen d'ajuster aux charges familiales un système global de sécurité sociale basé sur des assurances contributoires et obligatoires. Le rapport n'avait pas eu d'influence directe sur le cabinet King, qui n'adoptera jamais le système global mais uniquement les allocations.

La mesure sociale apparait aussi comme le moyen le plus facile d'éviter une dépression d'après-guerre. Dans l'optique du gouvernement de plus en plus gagné aux idées keynésiennes, la distribution d'un supplément de revenu aux ménages pauvres permettrait d'augmenter suffisamment la consommation pour empêcher un ralentissement de l'économie. De plus, devant l'incertitude des marchés étrangers d'aprèsguerre, Mackenzie King voit dans les allocations un moyen de renforcer le marché domestique: «Je dirai que presque tout l'argent qui sera dépensé sour forme d'allocations familiales servira à acheter les denrées produites ou fabriquées dans notre pays», déclare-t-il en Chambre?

A ces facteurs principaux s'ajoute une bonne dose d'électoralisme, le scrutin fédéral étant attendu en juillet 1945, dirigé à la fois vers la clientèle menaçante du jeune parti «socialiste», le CCF, et vers les Canadiens français échaudés par la conscription et chez qui l'idée des allocations familiales avait atteint une certaine popularité dans les années 1927-1932.

Le père Léon Lebel, s.j., aumônier général de l'Union catholique des cultivateurs canadiens de 1929 à 1934 et de 1937 à 1952, avait en effet donné plusieurs conférences et publié quelques brochures sur les allocations familiales et l'urgence de les instaurer, au cours de cette période. Le régime proposé était financé conjointement par le fédéral, le provincial, les municipalités, les employeurs, les célibataires et les hommes mariés sans enfants. Il visait principalement la réduction des injustices du système salarial pour les familles nombreuses. En 1927, le Comité des relations industrielles de la Chambre des Communes avait trouvé le mouvement d'opinion assez important pour faire comparaitre le père Lebel à ses audiences. De plus, la Commission Montpetit de 1930-1933 avait relevé «une adhésion plutôt sympathique au régime» à travers la province. Enfin, les syndicats catholiques avaient donné leur adhésion au projet à cette époque, alors que la plupart des autres centrales ont désapprouvé le principe jusque avant la guerre par peur que les allocations servent d'alibi au maintien de bas salaires. Mais la Commission Montpetit avait rejeté l'idée des allocations parce que, disait-elle, le régime correspondait à la situation européenne où les salaires étaient peu élevés, qu'il commandait l'intervention de l'État et qu'il était coûteux. Il aurait aussi placé le Québec en «situation désavantageuse par rapport aux autres provinces» et encouragé l'exode

7 Canada, Débats de la Chambre des Communes. 1944, V: 5534. 
rural, toujours selon les commissaires. De la même façon, Esdras Minville, que la Commission Rowell-Sirois avait chargé, à la veille de la guerre, d'enquêter sur le régime social de la province de Québec, n'avait pas proposé d'allocations familiales. Ses réticences vis-à-vis des pensions gratuites, de l'étatisme et de l'intervention fédérale en matière de bien-être avaient vraisemblablement motivé son silence sur la question ${ }^{8}$.

De plus, les allocations familiales permettent au gouvernement central d'accroître sa présence dans le secteur du bien-être que la constitution réserve en principe aux provinces et aux municipalités. Cette règle avait obligé le fédéral à demander une modification de la constitution pour promulguer sa Loi de l'assurance-chômage en 1940. En 1944, le gouvernement King contourne l'obligation en échafaudant un système d'allocations familiales non-contributoires et facultatives, et en invoquant le pouvoir de dépenser du fédéral. Tout se passe comme si les allocations lui fournissaient une occasion supplémentaire de dépenser qui allait le placer en position de force pour les négociations fédérale-provinciales à venir sur la fiscalité d'après-guerre. Que les allocations aient été pensées dans ce but n'est pas certain, mais d'avril 1945 à mars 1946, les allocations composeront 16\% des dépenses civiles ordinaires du fédéral ${ }^{9}$. Quand le gouvernement King proposera de s'approprier l'ensemble des pouvoirs d'imposition, à la conférence fédérale-provinciale du rétablissement d'août 1945, il invoquera les coûts du «vaste programme de développement national et de bien-être public» qu'il doit entreprendre et rappellera le précédent de la Loi des allocations familiales ${ }^{10}$.

\section{2 - L'État fédéral et les responsabilités parentales: aide ou appropriation?}

Au cours des brefs délais qui précèdent l'adoption de la législation, Mackenzie King énonce des objectifs vis-à-vis des familles: l'État doit aider celles-ci à élever leurs enfants et doit protéger les enfants quelle que soit la conjoncture économique. Dans «l'ordre nouveau» qui s'imposera après la guerre, les enfants constitueront, dit-il, un avantage pour le pays, un capital humain dont la santé et le bien-être seront essentiels à l'économie, la défense et la survie de la nation ${ }^{11}$. Ainsi les périodes de guerre ramènent-elles toujours au centre des préoccupations

8 Québec, Commission des assurances sociales de Québec. Troisième rapport (Québec, 1933), 90, 107, 108 et Esdras Minville, La législation ouvrière dans la province de Québec (Ottawa, Imprimeur du Roi, 1939), 74, étude préparée pour la Commission Royale des Relations entre le Dominion et les Provinces.

9 Dominion Bureau of Statistics, The Canada Year Book. 1947 (Ottawa, Imprimeur du Roi, 1947), 956-957.

10 Canada, Conférence fédérale-provinciale du rétablissement, août 1945, 30, 53.

11 Débats analysés par François-Albert Angers, «Les allocations familiales fédérales de 1944», L'Actualité économique, 3 (juin-juillet 1945): 228-262 et Canada, Débats..., op. cit., 5531 . 
politiques l'importance de l'amélioration de la vie familiale et celle du rôle maternel, en transformant toute question de main-d'oeuvre en une affaire d'urgence et en donnant une valeur renouvelée aux vies humaines que les conflits menacent ${ }^{12}$.

De cette façon, le gouvernement King se démarque clairement de l'idéologie qui confie la charge unique des enfants à leurs parents. Mais l'État providence naissant s'approprie-t-il du même coup une partie du rôle de la famille et de l'école dans la définition de l'enfance, comme l'avancent les historiens Rooke et Schnell ${ }^{13}$ ? La présomption de contrôle assortie aux allocations permet-elle une extension, au moyen de l'appareil d'État, des possibilités de contrôle du comportement et du développement des travailleurs canadiens par les classes moyennes, comme le suggère Strong-Boag pour les lois provinciales des mères nécessiteuses des années 1916-1939? Ou enfin, «la majorité anglo-protestante d'Ottawa [a-t-elle] apposé dans la loi la marque de sa conception propre de la famille et de l'organisation familiale, en faisant le minimum de concessions aux idées de la minorité catholique et française sur le sujet», comme le prétendait l'économiste François-Albert Angers en $1945^{14}$ ?

\section{1 - État et familles: une intrusion faiblement contestée}

Plusieurs politiciens et intellectuels contemporains, canadiens et québécois, craignent l'intrusion de l'État dans les familles. Ils dénoncent les pouvoirs dangereux et arbitraires du fédéral sur l'éducation que les parents donnent à leurs enfants, la diminution conséquente des responsabilités parentales et l'attitude de suspicion dont la loi fait preuve à l'égard des familles ${ }^{15}$. Les opposants s'en prennent surtout à l'article de la loi qui stipule que «la personne qui reçoit l'allocation doit l'affecter exclusivement à l'entretien, au soin, à la formation, à l'instruction et à l'avancement de l'enfant», et "si le Ministre ou le fonctionnaire que les règlements autorisent à cet égard est convaincu que l'allocation n'est pas ainsi affectée, le versement en doit être discontinué ou fait à quelque autre personne ou organisme» ${ }^{16}$.

12 Veronica Strong-Boag, «Wages for Housework: Mother's Allowances and the Beginning of Social Security in Canada», Journal of Canadian Studies/Revue d'études canadiennes, 14,1 (Spring 1979): 24-25.

P. T. Rooke et R. L. Schnell, Discarding the Asylum. From Child Rescue to the Welfare State in English Canada (1800-1950) (Boston, University Press of America, 1983), 412.

${ }_{14}$ V. Strong-Boag, op. cit., 28; F.-A. Angers, op. cit., 248.

15 Léon Lebel, s.j., «Après deux ans d'allocations familiales», Relations, 7,80 (août 1947): 228; la conservatrice Charlotte Whitton, auparavant directrice du Conseil canadien de bien-être et représentante du Canada au Comité sur les questions sociales de la Société des nations, citée par A. M. Willms, Setting Up Family Allowances, 1944-45, thèse de M. A. (Administration publique), Carleton University, 1962, 228; Éditorial du Devoir, 11 août 1944, présenté par Micheline Carrier, Les craintes de l'élite clérico-nationaliste face à la loi des allocations familiales (194445). Essai d'interprétation d'une idéologie conservatrice, travail inédit (Histoire), Université Laval, août 1976.

16 «Loi de 1944 sur les allocations familiales», op. cit., article 5. 
Le Directeur des allocations familiales peut en effet exiger des parents des informations à l'égard de l'usage qui est fait de l'allocation et ordonner une enquête à ce sujet ${ }^{17}$. Au Québec, les cas de mauvaise utilisation, allégués par l'administration des allocations elle-même, par des agences privées et publiques consacrées au bien-être de l'enfance, ou par des institutions, individus ou membres de la famille, sont examinés sur le terrain par l'équipe de sept travailleurs sociaux et d'une dizaine de commis de la section bien-être du Bureau régional. Quelques cas sont confiés à des agences privées qui reçoivent $5,00 \$$ par enquête. Quand l'allégation s'avère fondée, ces investigations exhaustives, chacune d'entre elles donnant lieu à la visite de six personnes en moyenne ${ }^{18}$, mènent au versement de l'allocation à un tiers parti, à une agence ou à un citoyen digne de confiance. Autrement, quand la maladie, la mort ou la séparation changent la configuration familiale, un autre bénéficiaire est désigné dans la famille. Enfin, la famille peut être référée aux travailleurs sociaux d'une agence de bien-être locale ou recevoir des recommandations spéciales quand l'enquêteur découvre un «piètre sens de l'administration du foyer». On peut alors exiger de la bénéficiaire, pendant un certain temps, un rapport périodique sur l'affectation de l'allocation ${ }^{19}$.

Le programme devient donc un moyen privilégié pour les travailleurs sociaux de découvrir les situations jugées problématiques... pour le meilleur, à croire L. H. Senez, responsable de la section du bien-être au Québec, qui affirme que «[a] government such as ours being in the position to «peep in» such a large number of families and put «our thumb» on so many unhappy situations, can do a lot to assist Canadians to live more useful and satisfying life ${ }^{20}$.

La réaction des familles au nouveau pouvoir que s'attribue le fédéral est très difficile à cerner. Il est clair cependant qu'une très faible proportion d'entre elles est soupçonnée de mauvaise utilisation. Le tableau 1 montre que cette proportion atteint son maximum en 19501951 quand près d'une famille sur 700 reçoit la visite d'un enquêteur.

Parmi elles, moins d'une famille sur cinq voit l'administration de l'allocation confiée à un tiers parti. Ce qui fera dire en 1947 à Léon Lebel que «jusqu'ici, les fonctionnaires chargés de l'application de la

17 «Règlement sur les allocations familiales», op. cit., 3756.

18 Rapport annuel du Service de bien-être du bureau régional de Québec, 1950-51 (à l'avenir $R A S B E B R Q$ ), 5, Archives publiques du Canada, RG 29, vol. 1283. Le chiffre est tiré d'une enquête sur les activités du service de bien-être menée au cours du mois de juillet 1950 .

19 Welfare Statistical Report. Definitions and Comments. Revised, June 1950, APC, RG 29, vol. 1933, R233-100-1/52; RASBEBRQ, 1949-50, 18, APC, RG 29, vol. 1283.

20 RASBEBRQ, 1954-55, 5, APC, RG 29, vol. 1283. De plus, les bureaux régionaux agissent souvent comme des agences d'information sur les services sociaux disponibles. R. H. Parkinson (responsable national des services de bien-être), «Ten Years of Family Allowances», Canadians Welfare, 21,4 (November 1955): 195-200. 


\section{TABLEAU 1}

Enquêtes sur la mauvaise utilisation des allocations familiales 1949-1955. Province de Québec

\begin{tabular}{|c|c|c|c|c|c|c|}
\hline Année & $1948-49$ & $1949-50$ & $1950-51$ & $1951-52$ & $1952-53$ & $1954-55$ \\
\hline $\begin{array}{l}\text { Nombre de } \\
\text { familles } \\
\text { bénéficiaires }\end{array}$ & 488263 & 507727 & 525358 & 542651 & 564219 & 605916 \\
\hline $\begin{array}{l}\text { Mauvaise } \\
\text { utilisation } \\
\text { alléguée }\end{array}$ & $\begin{array}{c}375 \\
(0,08 \%)\end{array}$ & $\begin{array}{c}539 \\
(0,1 \%)\end{array}$ & $\begin{array}{c}789 \\
(0,15 \%)\end{array}$ & $\begin{array}{c}764 \\
(0,14 \%)\end{array}$ & $\begin{array}{c}498 \\
(0,09 \%)\end{array}$ & $\begin{array}{c}433 \\
(0,07 \%)\end{array}$ \\
\hline $\begin{array}{l}\text { Mauvaise } \\
\text { utilisation } \\
\text { effective }\end{array}$ & - & - & 135 & 95 & 52 & 76 \\
\hline $\begin{array}{l}\text { Allocation } \\
\text { versée à un } \\
\text { tiers parti }\end{array}$ & 17 & 33 & 58 & 22 & - & 36 \\
\hline $\begin{array}{l}\text { Changement de } \\
\text { bénéficiaire }\end{array}$ & - & 18 & 21 & 43 & - & 13 \\
\hline $\begin{array}{l}\text { Recommandations } \\
\text { spéciales }\end{array}$ & - & 79 & 132 & 212 & - & 71 \\
\hline Non changement & - & 408 & 578 & 487 & - & 313 \\
\hline $\begin{array}{l}\text { Référés à agences } \\
\text { sociales (inclus } \\
\text { dans non-changement) }\end{array}$ & - & 14 & 12 & 11 & - & - \\
\hline
\end{tabular}

Sources: Rapport annuel du Bureau régional de Québec, et Rapport annuel du service de bienêtre du Bureau régional de Québec, pour les années mentionnées, le rapport de 1953-1954 manquant, APC, RG 29, Vol. 1283; Rapport annuel du ministère de la Santé nationale et du Bienêtre social, Ottawa, Imprimeur du roi, pour les années mentionnées. Les statistiques d'avant 19481949 n'ont pas été trouvées. La précision des statistiques varie d'une année à l'autre. Le trait (-) indique que l'information n'est pas disponible.

loi ont su respecter l'autonomie des familles» ${ }^{21}$. Mais les familles touchées perçoivent-elles l'action des travailleurs sociaux comme une aide ou une usurpation de leurs responsabilités?

En 1951, deux cent quatorze mères de Québec échantillonnées d'après le montant des prestations qu'elles reçoivent sont invitées à se prononcer sur le régime des allocations et à suggérer des améliorations, par des chercheurs universitaires qui prennent soin de bien se distinguer des enquêteurs gouvernementaux. Or aucune d'entre elles ne désapprouve le programme en invoquant le fait que les responsabilités paren-

21 Léon Lebel, op. cit., 230. Lebel souhaite néanmoins la modification de ce volet de la loi au cas où «un fonctionnaire trop zélé... [exige] qu'on lui prouve, documents en main, que l'argent a réellement été employé exclusivement... au bénéfice des enfants», mais il apprécie encore, en 1951 , la «discrétion» du gouvernement dans les faits. "Les allocations familiales au Canada», Relations, 11,122 (février 1951): 47-50. 
tales sont usurpées ${ }^{22}$. La même absence de critique se retrouve dans une étude menée dans les fermes du comté de Nicolet, en 1948, auprès de 115 familles $^{23}$. Par ailleurs, parmi 79 mères d'enfants d'âge préscolaire du sud-ouest de Montréal au revenu modeste, rencontrées au printemps 1947, seulement $11 \%$ sont opposées aux allocations ou sceptiques vis-à-vis du programme, soit parce qu'elles désapprouvent l'interférence du gouvernement, soit parce que les allocations ne sont pas d'un grand intérêt. Dans ces derniers cas, soit $4 \%$ du total, les conjoints manifestent souvent des opinions divergentes sur la question, l'homme affirmant qu'il peut élever ses enfants seul et suspectant le gouvernement de ne pas lui donner de l'argent pour rien ${ }^{24}$. Ces indications rejoignent celles d'une enquête plus vaste menée en Alberta et en Saskatchewan auprès de 416 familles rurales: sur les six familles opposées au système, trois seulement ont expliqué qu'elles pouvaient elles-mêmes prendre soin de leurs enfants ${ }^{25}$.

Les mères québécoises interviewées ne sont probablement pas celles que les enquêteurs du Bureau régional ont importunées. Reste qu'aucune d'entre celles-là ne paraît avoir été témoin, dans le voisinage ou la parenté, d'une intrusion de l'État digne de mention. Peut-être ontelles eu peur de critiquer le programme. Une anecdote racontée par Thérèse Légaré, travailleuse sociale chargée par le «Cercle du Terroir» de Montréal d'étudier les effets des allocations dans la région de GaspéNord, le laisse entendre:

A l'occasion de notre enquête, écrit-elle, le bruit s'est répandu que c'était une enquêteuse des allocations qui visitait les familles et qu'elle «enlèverait les allocations» aux familles qui ne les utilisaient pas pour leurs enfants... Ceci a évidemment influencé leurs réponses. ${ }^{26}$

Enfin, les femmes montréalaises qui s'excusent, lors de certaines entrevues, d'utiliser les allocations pour la nourriture en les ajoutant au revenu familial, font entrevoir une pression plus sournoise sur l'économie domestique.

22 Maurice Tremblay, Albert Faucher et J.-C. Falardeau, Family Allowances in Québec City. Report of a Study in the Faculty of Social Sciences of Laval University (Québec, 1951), translated by the Department of National Health and Welfare, 48, APC RG 29, R233/100-63-2.

${ }_{23}$ M. A. Macnaughton et G. Laflèche, Preliminary Report on Distribution and Use of Family Allowances Payments in Nicolet County, Québec, 1947-48 (Ottawa, Economic Division, Marketing Service, Department of Agriculture, 1936), 19-23, APC, RG 29, R233/105-13/5.

${ }_{24}$ Blishen et al., op. cit., 96-105.

25 M. A. Macnaughton et J. M. Mann, Distribution and Use of Family Allowance Payments in Three Areas of the Prairie Provinces. Supplément à M. A. Macnaughton et M. E. Andal, Changes in Farm Family Living in Three Areas of the Prairie Provinces from 1942-43 to 1947 (Ottawa, Economics Division, Marketing Service, Department of Agriculture and Department of National Health and Welfare in cooperation with the Universities of Alberta and Saskatchewan, King's Printer, 1949), 91, publication 815, Technical Bulletin 69.

26 Légaré, op. cit., 9, 132. 
Many women seemed to feel this might not be a way which the family allowances were supposed to be used, rapportent Blishen, Cawley et Pearson. Apparently, these attitudes arose from emphasis in family allowances publicity on the use of the money for children specifically. ${ }^{27}$

Bien que dans les faits le gouvernement n'oblige en aucun cas une bénéficiaire à dépenser pour les enfants séparément et exclusivement, la publicité peut inculquer à des parents des idéaux que la pauvreté empêche d'atteindre.

Bref, si les récipiendaires ont quelque objection à l'entrée potentielle de l'État dans la gestion des ressources familiales, elles se font beaucoup moins bruyantes que les élites politiques, religieuses ou intellectuelles. L'allocation d'une somme d'argent régulière est assez appréciée pour que les présomptions de contrôle qui s'y joignent soient oubliées par la grande majorité du public. L'Institut canadien de l'opinion publique relève une approbation phénoménale du programme, qui s'accroît de 49\% à 90\% des Canadiens de 1943 à 1955, une des majorités les plus spectaculaires rapportées par l'Institut sur les questions nationales et internationales depuis 1940. Les Québécois témoignent d'un enthousiasme record, évalué à $96 \%{ }^{28}$, probablement attribuable aux bas revenus et salaires caractéristiques du marché du travail québécois, au nombre important d'enfants par famille de même qu'à la faible proportion de femmes mariées travaillant hors du foyer.

Au pouvoir légal d'entrer dans les foyers s'ajoute le «vaste programme de propagande éducative [qui] vise... à gagner et à soutenir la compréhension et l'appui du public envers les mesures qui ont pour objet d'élever les normes d'hygiène et de bien-être social au pays ${ }^{29}$. Le programme des allocations familiales fournit un canal privilégié de diffusion de ces idées. En 1949-1950, on annonce, dans les enveloppes contenant les chèques, que l'opuscule intitulé Nos enfants sur les allocations, leur emploi, le budget familial, l'hygiène et l'éducation des enfants, est disponible à qui en fait la demande ${ }^{30}$. Une projection fixe, «Parlons des allocations familiales», un film intitulé «Peppo», des feuillets insérés dans les chèques, des affiches et des étalages destinés aux conférences et aux expositions régionales illustrent la manière la plus sage de dépenser les allocations ${ }^{31}$. L'État tente ainsi de diffuser au

27 Blishen et al., op. cit., 82.

28 Canadian Institute of Public Opinion, «Gallup Poll of Canada. 90 p.c. in Canada in Favor of Family Allowances», Toronto Star, 12 mars 1955. De plus, dans le comté de Nicolet, seulement une famille sur 66 éligibles au programme ne s'est pas inscrite. Laflèche et Macnaughton, op. cit., 4.

Rapport annuel du Ministère de la santé nationale et du bien-être social, 1950-51 (Ottawa, Imprimeur du Roi, 1951), 92 (à l'avenir RAMSNBES).

30 RAMSNBES, 1949-50, 94.

31 RAMSNBES, 1945-46, 90; 1945-47, 94; 1947-48, 130; 1950-51, 92. 
plus grand nombre ses vues en matière d'éducation des enfants. Mais, d'un autre côté, la demande pour ce type de renseignements est forte: en un an, 375000 bénéficiaires auront demandé qu'on leur envoie Nos enfants, soit le quart des mères des provinces sollicitées ${ }^{32}$. Et que dire de la popularité du livre du docteur Ernest Couture, La mère canadienne et son enfant, publié en 1940 et revisé en 1948-1949, annoncé avec les chèques lui aussi, dont le ministre Paul Martin autographie le millionième exemplaire au cours de l'année 1948-1949? A la veille d'une autre révision, en 1953, le ministère annonce que quatre millions de copies des versions antérieures ont été distribuées ${ }^{33}$. Une soif intense d'informations de ce genre se répand dans l'après-guerre. Elles ne font pas encore partie du savoir commun, comme aujourd'hui, grâce à l'intensification des communications et à l'expansion des services médicaux. De ce point de vue, on est tenté de conclure, comme Madison, qu'avec les allocations familiales, «the quality of childrearing may have improved in many homes because parents... have been reached by information about modern child welfare philosophy and community ressources for children» ${ }^{34}$.

\section{2 - Classes moyennes et familles à faible revenu: une plus grande confiance dans la population}

Il est vrai que les allocations offrent aux classes moyennes une possibilité supplémentaire de contrôle du mode de vie des travailleurs dans la mesure, d'une part, où le Bureau régional de Québec emploie des travailleurs sociaux. Or à sa naissance, à la fin des années 1930, le service social canadien-français, d'après l'historien du travail social Lionel Groulx,

n'est plus la charité au sens traditionnel mais plutôt la regénération sociale et morale qui s'exprime par la restauration de l'ordre familial, sa moralisation - qu'on peut interpréter comme une condamnation du mode de vie populaire — , sa rechristianisation, et par la pacification des relations entre les classes. [Et ce] nouveau dispositif de contrôle est appuyé par les groupes sociaux dominants de l'époque, la bourgeoisie et le haut-clergé. ${ }^{35}$

Ajoutons que l'exemption d'impôt pour enfants, instituée en 1918 , avait déjà permis à l'État d'aider les familles plus à l'aise à élever leurs enfants sans y joindre de semblables mesures de vérification de l'argent

32 RAMSNBES, 1950-51, 81. La distribution n'est pas terminée au Manitoba, en Saskatchewan et au Nouveau-Brunswick au moment de la rédaction du rapport.

33 RAMSNBES, 1948-49, RAMSNBES, 1952-53, 111. Légaré découvre même que jusqu'à St-Octave de l'Avenir, village de colonisation récente où il n'y a même pas de médecin, plusieurs mères consultent le volume. $O p$. cit., 74.

34 Bernice Madison, «Canadian Family Allowances and their Major Social Implications», Journal of Marriage and the Family, 26,2 (May 1964): 141

35 Lionel Groulx, «Le service social au Canada français. Ses énoncés et son rôle», Revue '83/Review' $83,148,150$. 
concédé. Si les allocations sont vues par plusieurs législateurs comme une extension du principe de l'exemption à tous les ménages avec enfants, quels que soient leurs revenus, elles révèlent ainsi une moindre confiance à l'égard des familles pauvres.

De plus, la loi souscrit sous plusieurs aspects à un idéal familial petit-bourgeois semblable à celui des travailleurs sociaux. Sans avoir adopté de politique familiale explicite, en effet, les gouvernements canadiens ont toujours pris des mesures dispersées touchant les familles, desquelles il est possible de dégager un ensemble d'intentions pour ces familles. Cette absence d'unité, qui paraît être le propre des pays anglosaxons, agit selon Kitchen comme un écran au fait que «historically, governments have seen the manipulation of family life as a way to achieve and maintain broader economic and political control» ${ }^{36}$. Dans le cas des allocations familiales, les législateurs souhaitent avant tout la dépendance prolongée des enfants, le maintien de la cellule familiale comme le meilleur milieu d'éducation, et la permanence de la mère à la maison, ce qui suppose un salaire unique pour la famille. Le versement du chèque à la mère, par exemple, même s'il représente une reconnaissance de la contribution des femmes au foyer, peut être associé à la campagne du milieu des années 1940 pour encourager les femmes à rester à la maison. A cette époque en effet, le gouvernement fédéral entend «résoudre partiellement le problème [de la fermeture des usines de guerre] par le retour au foyer des femmes mariées, dont la proportion sur le marché du travail est passée de $10 \%$ en 1939 à environ $35 \%$ en $1944{ }^{37}$. De plus, le taux décroissant désavantage les familles nombreuses qui se retrouvent en plus grande proportion parmi les classes pauvres, ce qui expliquerait d'ailleurs l'indignation du chef du CCF à ce propos, lors des débats sur la loi ${ }^{38}$.

Cette vision de l'impérialisme culturel des classes aisées mérite cependant d'être fortement nuancée. En discutant d'abord l'un des objectifs les plus chers des législateurs et des travailleurs sociaux: la promotion de la famille naturelle en tant que meilleur environnement pour le développement de l'enfant. Dans ce but, la loi empêche tout paiement à une institution. Elle accorde l'allocation aux mères des enfants placés si les enfants sont entretenus par leurs parents pour un minimum de 5,00\$ par mois. En l'absence d'une telle éventualité, elle ne permet que des versements à des agences de placement reconnues

36 Kitchen, op. cit. (1984): 186-187.

37 Geneviève Auger et Raymonde Lamothe, De la poêle à frire à la ligne de feu. La vie quotidienne des Québécoises pendant la guerre '39-'45 (Montréal, Boréal Express, 1981), 160. L'établissement des allocations aux mères nécessiteuses, par les gouvernements provinciaux du pays, dans la décennie suivant la Première Guerre, constitue un phénomène similaire.

38 Canada, Débats..., op. cit., 5544-5546. «A mesure que la famille augmente, dit-il, le problème tend à s'aggraver plutôt qu'à diminuer... le programme ne peut manquer de créer des distinctions injustes de traitement envers les familles nombreuses». 
qui prennent les enfants des orphelinats sous leur tutelle, c'est-à-dire celles qui ont un service d'adoption et qui rencontrent les critères du travail social moderne. On veut ainsi que tous les enfants aient le plus de chances possibles d'être adoptés ou placés en foyers d'accueil ${ }^{39}$.

Ce dessein explicite d'affaiblissement de la pratique traditionnelle du placement en institution rencontre les aspirations des familles les moins fortunées. En 1944, en effet, la Commission d'assurance-maladie de Québec avait relevé le fait que, dans les institutions publiques, une très faible proportion seulement des enfants étaient orphelins de père et de mère ${ }^{40}$. Un peu plus tard, une enquête sur la mise en institution des enfants de mères nécessiteuses menée dans la région de Québec entre 1948 et 1953 confirme l'existence du curieux phénomène:

$20 \%$ des enfants placés en institution [sont] des enfants de mères bénéficiaires d'allocations à titre de mères nécessiteuses. Dans cette seule région, 1000 enfants auraient pu rester dans leur famille et ont été placés dans des institutions à cause de l'insuffisance de - revenu de la mère. ${ }^{41}$

Le recours à l'institution par les parents de ces enfants n'est utilisé que comme étape ultime des stratégies économiques des familles faisant face à des difficultés insolvables, comme l'illustrent ces constats de L. H. Senez:

The institutions report that since the event of Family Allowances, the parents are happy and proud to be able to better care for their children away from home. Prior to 1945 children mostly stayed in the institutions during the Christmas and the Summer vacations. Now the institutions are literally empty. Only the orphans and abandoned children remain in the institutions during vacation time and that may represent only $10 \%$. The institutions of Giffard and Levis have reported that before 1946, only 50 children out of 600 would spent the two months summer holidays in their families. Now only 50 remain in the institution, the others can go home. ${ }^{42}$

Pour évaluer l'autonomie des familles, il convient ensuite d'examiner le niveau de confiance et de latitude accordé aux parents par la

39 «Loi de 1944 sur les allocations familiales, op. cit., article 2; «Règlements sur les allocations familiales», op . cit., 3755. Pour l'adhésion de la profession des travailleurs sociaux au principe du maintien des liens familiaux, voir Strong-Boag, op. cit., 32.

${ }_{40}$ Québec, Premier Rapport de la Commission d'assurance-maladie de Québec sur le problème des garderies et de la protection de l' enfance (Québec, 1944), 8 (Commission Garneau).

${ }_{41}$ Québec, Rapport de la Commission royale d'enquête sur les problèmes constitutionnels, Vol. III, Tome I (Québec, Eugène Doucet Imprimeur, 1956), 81.

${ }_{42}$ RASBEBRQ, 1949-50, op. cit., 13-14. Plus globalement que les allocations familiales, la prospérité générale des années d'après-guerre est probablement responsable de ce resserrement des liens familiaux. Pour l'époque précédente, la même attitude vis-à-vis des orphelinats a été démontrée par Bettina Bradbury. «The Fragmented Family: Family Strategies in the Face of Death, Illness and Poverty, Montreal, 1860-1885», in Joy Parr, ed., Childhood and Family in Canadian History (Toronto, McClelland \& Stewart, 1982), 109-128 et Joy Parr, pour les orphelinats britanniques, Labouring Children. British Immigrant Apprentices to Canada, 1869-1929 (Montreal, London, McGill-Queen's University Press, Croom-Helms, 1980), 62. 
loi. En avançant l'idée de l'entretien comme critère de sélection des parents méritants, les législateurs tentent de s'éloigner de l'approche subjective de la charité, en tenant compte de l'avis de la population assistée:

We soon found, rappelle en 1948 George Davidson, sous-ministre de la Santé nationale et du Bien-être social, aux directeurs des services de bien-être du Canada, an increasing resistance on the part of the public to the idea that any person, social worker or not, should presume to decide who is a deserving case and who isn't a deserving case. We got to the stage where people began to demand that legislation be written down in specific terms to provide as a matter of right certain benefits to people under clearly defined conditions that were prescribed in the law rather than left to the judgment of some individual. Our legislation since that time has been a mixture of those two things. ${ }^{43}$

Ainsi délivré des «means tests» et des méthodes discrétionnaires des années 1930, le public s'opposera de plus en plus aux formules à saveur ancienne $^{44}$. A l'échelle individuelle, cette attitude est bien illustrée par le cas d'une femme dont le mari souffre de maladie mentale, rapporté en 1947 par une étudiante en service social de l'Université McGill:

Lou applied for Needy Mother's Allowance, écrit-elle, but the uncertainty of the duration of Bob's illness made her ineligible... Family Allowances of \$24 was the only income of which she was certain. As she discussed a budget, the fact that she had it, and had a right to it, seemed to restore some of her self-assurance... ${ }^{45}$

On rencontre donc, en suivant les débats entourant l'adoption de la loi, un étrange paradoxe: ceux qui veulent préserver la famille des incursions de l'Etat en conservant des programmes non-universels sont aussi ceux qui feraient le moins confiance à la mère pour la dépense des allocations. Pendant que François-Albert Angers a peur que les familles se prélassent dans la trop paternelle providence de l'État et qu'elles soient gagnées par l'esprit de lucre, pendant que les Conservateurs craignent que les chèques soient mal dépensés, King a beau jeu de proclamer que «Liberals [are] willing to trust people whereas Tories always want to manage their affairs for them ${ }^{46}$.

Cependant, il existe trois groupes de Canadiens à qui cette protection accrue n'est distribuée qu'à la goutte. Il s'agit d'une part des Inuits et des «nomades», qui reçoivent tous leurs paiements en nature jusqu'en

43 Maintenance as an Eligibility Factor. Dr Davidson's Remark at Supervisors' Conference, with Introductory Discussion, Ottawa, March 23-25, 1948, APC, RG 29, vol. 1934, R233/ $100-6 / 25$.

44 Dennis Guest, The Emergence of Social Security in Canada (Vancouver, University of British Columbia Press, 1981), 143. $27-28$.

Agnes Tennant, «Family Allowances Story», The Social Worker, 15,3 (February 1947):

46 Angers, op. cit.; Kitchen, op. cit. (1981): 45. Léon Lebel se présente comme une exception à cette règle. 
$1953^{47}$. D'autre part, dans le cas des Amérindiens, le ministère distribue des primes soit en nature, soit en argent, ou encore sous la supervision des agents des Indiens, «selon le degré de responsabilité exercé par les parents» ${ }^{48}$. Quand en $1947-1948$ une proportion record de $75 \%$ des mères reçoivent leur chèque directement, on remarque paternellement dans le rapport annuel que «les autorités chargées de l'application de la loi ont jugé qu'un plus grand nombre de familles indiennes pouvaient utiliser à bon escient les allocations familiales». Enfin, la loi réserve une clause de résidence de trois ans aux immigrants. La discrimination sera atténuée en 1949 quand on réduira la période à un an. «Ces versements plus hâtifs, reconnaîtra alors le ministère, ont mis plus à l'aise maintes nouvelles familles canadiennes à une période où leurs ressources se trouvaient limitées et leurs besoins censés être considérables.» ${ }^{49}$

Pour résumer, si la loi comporte des clauses par lesquelles les classes dirigeantes peuvent influencer la composition et la structure des familles pauvres, amérindiennes, inuits et immigrantes, elle témoigne avant tout d'une confiance inégalée envers la famille comme lieu d'éducation des enfants et comme agent de leur bien-être.

\subsection{Législateurs canadiens-anglais et familles québécoises: des allocations et non des discours sur l'autonomie}

Il nous reste, au terme de cet article, à chercher en quoi les conceptions anglo-protestante et franco-catholique de la famille s'opposeraient à l'occasion de l'application de la loi.

La question qui soulève le plus de tensions entre les deux groupes est sans contredit celle de l'encouragement aux familles nombreuses. Alors que le Québec compte la plus grande proportion de familles de grande dimension, le gouvernement King décide de diminuer le montant de l'allocation après le cinquième enfant ${ }^{50}$. Il invoque le fait que

47 A partir de cette date, de nouveaux règlements permettent le versement direct aux parents sur recommandation du ministère des Mines et ressources et du développement économique. RAMSNBES, 1952-53, 89; "Règlements des allocations familiales», op . cit., 3757-3758. «Nomade» signifie «une personne de sang indien ou esquimau mêlé, résidant dans les Territoires du NordOuest ou le Territoire du Yukon, qui n'est pas un Esquimau ou un Indien, mais qui vit à la façon des Esquimaux ou des Indiens. Cette distinction est abandonnée en 1948, car elle donne une image de racisme, d'après Willms, op. cit.

48 RAMSNBES, 1949-50, 150. De plus, la procédure de demande est alourdie pour tous les autochtones par des procédures de vérification supplémentaires. En 1955, suite à la pression de bandes indiennes, elle sera normalisée. RAMSNBES, 1954-55, 115.

49 «Loi de 1944 sur les allocations familiales», op. cit., article 2 . La clause de résidence étonne quand on sait que par ailleurs les promoteurs de la loi y voyaient un encouragement à l'immigration. Davidson, 1944, cité par Blishen et al., op. cit., 26.

${ }_{50}$ Le nombre moyen d'enfants par famille dépasse au Québec celui de toutes les autres provinces. Il s'élève à 2,4 et la moyenne canadienne est de 1,9. Recensement du Canada. 194I, V: 178-237; «Loi de 1944 sur les allocations familiales», op. cit., article 3. La réduction est fixée à un dollar pour le cinquième enfant, deux dollars pour les sixième et septième, trois dollars pour les enfants suivants, alors que les primes se chiffrent à cinq, six, sept ou huit dollars selon que l'enfant a moins de 6 ans, 6 à 9 ans, 10 à 12 ans ou 13 à 15 ans respectivement. 
l'entretien des enfants coûte moins cher après le quatrième car ceux-ci héritent des vêtements, jouets et livres de leurs frères et soeurs plus âgés et qu'une jeune famille rencontre des dépenses de base qui ne se renouvelleront plus par la suite ${ }^{51}$. Ce principe était déjà inscrit dans le rapport Marsh, de même que dans son pendant britannique et inspirateur, le rapport Beveridge, et dans la loi des allocations aux dépendants de soldats. Ceci porte à croire qu'il n'est pas uniquement une concession aux pressions d'un groupe de Canadiens anglais qui s'opposent aux allocations parce qu'ils craignent que ces «baby bonus» avivent les antagonismes entre deux ethnies aux taux de natalité différents, déjà divisées sur la question de la conscription ${ }^{52}$. D'autres Canadiens anglais réfutent l'argument des baby bonus, cependant, et le parti libéral luimême s'applique à éviter que les débats sur la mesure sociale deviennent une question raciale ${ }^{53}$.

Le taux décroissant n'est donc pas une mesure prise délibérément par des Canadiens anglais contre les Canadiens français; King envisage même de retrouver la faveur du Québec au moyen des allocations. Mais il n'est pas non plus un encouragement à la famille nombreuse et à la natalité comme l'auraient voulu plusieurs promoteurs des allocations, Léon Lebel en tête ${ }^{54}$. En effet, les législateurs proviennent majoritairement du Canada anglais qui peut compter, pour sa croissance démographique, sur un important afflux d'immigrants, pour la venue desquels le gouvernement fédéral fournit déjà un effort soutenu. Ils s'inquiètent donc peu de la natalitées ${ }^{55}$. Jamais, dans les rapports annuels du ministère, n'essaie-t-on de lier l'augmentation du taux de natalité de l'aprèsguerre au versement des allocations. Au contraire, on insiste à plusieurs reprises pour démontrer l'invalidité de «l'argument autrefois émis,

51 Débats de la Chambre des Communes. 1944, V: 5526.

52 Willms, op. cit., 26-29. Parmi ces alarmistes, Charlotte Whitton et C. E. Silcox dont le plaidoyer s'intitule The Revenge of the Cradles (Toronto, Ryerson Press, 1945). Le taux décroissant vise aussi à calmer les tensions entre les ethnies. Lorsque l'accusation de favoritisme du programme des allocations à l'endroit du Québec est amenée en chambre, le ministre responsable de la loi, Brooke Claxton, allègue en effet que «cette mesure [le taux décroissant] a été préparée de façon à résoudre de manière la plus pratique possible le problème qui était d'accorder le même traitement à tous les Canadiens... [L'] Ontario et la Colombie-Britannique toucheront une plus forte somme par enfant, étant donné que la réduction qui a soulevé beaucoup d'opposition dans le Québec ne s'appliquera guère dans ces provinces». Débats..., op. cit., 5594. (1981): 48 .

«Arguments or Citizens», Liberty Magazine, Toronto, 14 avril 1945; Kitchen, op. cit.

54 Les arguments de Lebel sont repris en Chambre par quelques députés francophones libéraux et par les représentants du Bloc populaire. De plus, le CCF réclame un traitement équitable pour les familles nombreuses; Dorise Nielsen s'appuie sur son expérience de mère de famille pour dénoncer l'injustice et invoque même le faible niveau des salaires du Québec. Seuls Frédéric Dorion, le Bloc populaire et le député de Swift-Current confèrent des intentions natalistes à la loi. Débats..., op . cit., 5564-5568, 5592, 5598-5600, 5613. Dans l'ensemble, la loi n'a été ni conçue, ni votée, ni appliquée dans cet objectif cependant.

55 Au rang des exceptions chez les Anglophones, mentionnons Frederick Wright, du Bureau du Service municipal de Montréal, qui préface la traduction du pamphlet de Léon Lebel, The Problem of the Large Family in Canada. Its Solution. Family Allowances (Montréal, Georges, 1928), 2. 
d'après lequel les allocations familiales augmenteraient le nombre des familles nombreuses». Les allocations n'assumant qu'une partie de l'entretien des enfants, elles «ne sont probablement pas un stimulant par elles-mêmes», avance-t-on ${ }^{56}$. Ainsi, l'augmentation du taux de natalité des États-Unis qui n'ont pas adopté ce genre de loi sociale, est supérieure à celle du Canada $(28,2 \% \text { contre } 20,2 \% \text { de } 1939 \text { à } 1948)^{57}$. Pour expliquer le baby-boom, on invoque plutôt le retour des soldats, la fin de la crise du logement et l'abondance des emplois disponibles qui permettent de nouveaux établissements.

Néanmoins, le système de taux décroissants proposés par King heurte les idéaux catholiques et nationalistes de plein front. Pour le Canada français, en effet, l'évolution de la natalité représente un problème national. Avant 1961, son importance démographique dans l'ensemble du pays tient à sa surfécondité qui contrebalance l'effet de l'immigration dont il profite beaucoup moins que le Canada anglais ${ }^{58}$. Ce phénomène, comme le propose Armitage, «affect[s] social policy in Quebec and create[s] a greater conciousness of the need for family policies that provide for continuity in the national and cultural roles that the family has traditionally fulfilled» ${ }^{59}$. C'est à cette préoccupation nationale et à l'influence des sociologues européens qu'est due la précocité de la propagande en faveur des allocations dans la province. Dès 1927, Léon Lebel y avait vu le remède à l'émigration, à l'exode rural et à la «peur de l'enfant» dont commençaient à souffrir le Québec et le Canada $^{60}$. Dans cette optique nataliste, les versements proposés par Lebel commençaient au troisième enfant et augmentaient avec le rang de naissance ${ }^{61}$.

Une fois le principe du taux décroissant inscrit dans la loi, l'élite a non seulement des raisons morales et politiques de s'élever contre

$56 \quad$ RAMSNBES, 1946-47, 78-79; 1947-48, 100-101; 1948-49, 117.

57 RAMSNBES, $1947-48,101,176$. Il se peut que seules les particularités de la transition démographique du Québe:c soient responsables de la faible moyenne canadienne. Voir Jacques Henripin et Yves Peron, «La transition démographique dans la province de Québec», in Hubert Charbonneau, éd., La population du Québec: études rétrospectives (Montréal, Boréal Express, 1973), 39.

${ }_{58}$ Hervé Gauthier, Évolution démographique du Québec (Québec, Office de planification et de développement du Québec, 1977), xviii

59 Andrew Armitage, «Canada», in S. B. Kamerman et A. J. Kahn, ed., Family Policy: Governments and Families in Fourteen Countries (New York, Columbia University Press, 1978), 375.

60 Léon Lebel, s.j., Les allocations familiales. Solution du problème des familles nombreuses (Montréal, École Sociale Populaire, 1927), 22, 43, 45, 62. Ses informations sur la baisse de la natalité et l'émigration provenaient de l'Annuaire du Canada de 1925. Presque prophétiquement, Lebel osait même opposer à d'éventuelles objections des autres provinces le fait qu' «il est plus difficile de hausser le taux des naissances dans les régions à haute natalité que dans les autres. Sous ce rapport, ajoutait-il, les allocations favoriseraient davantage les régions à basse natalité... Ainsi les allocations familiales, en augmentant le taux de natalité chez nos compatriotes anglosaxons, sera tout à leur avantage bien compris et éloignera du pays le danger qu'il court de perdre sa physionomie britannique».

$61 \quad$ Ibid., 51; Léon Lebel, Le problème de la famille nombreuse. Sa solution. Les allocations familiales (Montréal, Imprimerie du «Devoir», 1928), 25. 
cette «prime à la famille restreinte» ${ }^{62}$, mais des raisons économiques, ces principes ayant une certaine résonance dans la réalité63. En 1944, en effet, Angers évalue à neuf millions de dollars le montant annuel supplémentaire que le Québec aurait reçu «si les familles nombreuses avaient été traitées sur le même pied que les familles restreintes» ${ }^{64}$. Dans les faits, quand en 1949 un amendement abolit le taux décroissant, la division des allocations familiales dépense $3500000 \$$ en réajustements au Québec pour l'année 1949-1950, soit 55\% du total des réajustements, alors que la province ne fournit que $32 \%$ des enfants éligibles $^{65}$. Les débats en Chambre ne permettent pas de comprendre pourquoi la clause qui avait semé une si grande controverse est retirée. Le ministre Paul Martin se contente d'expliquer que la modification tend «à remédier à certaines faiblesses de la loi primitive.... ${ }^{66}$

Duplessis reprend le principe d'une «sollicitude particulière aux familles nombreuses» lorsqu'il fait voter sa propre loi des allocations familiales, en février 1945, qui n'est en fait qu'un projet de négociations avec le fédéral «pour l'établissement d'un système d'allocations familiales conforme aux intérêts de la province» ${ }^{67}$. Les démarches du premier ministre en ce sens seront vaines. Il se repliera sur un ajustement des taux d'allocation aux mères nécessiteuses, mis en vigueur en septembre 1945, pour compenser la diminution des primes d'allocations familiales après le quatrième enfant. Mais les mères nécessiteuses représentent alors moins d'une bénéficiaire des allocations familiales sur trente-cinq ${ }^{68}$.

C'est auprès du groupe des familles nombreuses, le plus touché par ce biais canadien-anglais de la loi, que le mouvement de boycott des allocations promu par le Bloc populaire au nom de l'autonomie

62 Carrier, op. cit., 30-31. Le député Michaud, de Restigouche-Madawaska, va jusqu'à dénoncer les «effets psychologiques du taux décroissant» et l'insulte qui est faite aux enfants après le quatrième, que le fédéral ne considérerait que comme des portions d'enfants. Propos rapportés par Roy, op. cit., 24-25.

63 «Peut-être nulle part dans le monde, l'idéal catholique d'une nombreuse famille a-t-il été observé avec plus d'efficacité. Cet idéal fut renforcé par une propagande nationaliste vigoureuse en faveur de la revanche des berceaux et l'on peut difficilement douter du succès de ces idées». Henripin et Peron, op. cit., 41-42. Marie Lavigne a nuancé cette image de la fécondité des Québécoises cependant, en précisant qu' «une importante fraction des femmes ne se sont jamais mariées et [que] les Québécoises sont proportionnellement moins attirées vers le mariage que les autres Canadiennes. Entre 14\% et $15 \%$ [des femmes nées vers 1887, 1903 et 1913] ne se marient pas... [et] seulement une femme mariée sur cinq [a] dix enfants et plus». «Réflexions autour de la fertilité des Québécoises», in N. Fahmy-Eid et M. Dumont, éd., Maîtresses de maison, mấtresses d'école (Montréal, Boréal Express, 1983), 324.

64 Angers, op. cit., 261. Il utilise les données du recensement de 1931, ce qui explique en partie la différence avec la situation réelle exposée ci-après. L'adhésion à l'idéologie des familles nombreuses est probablement responsable de l'écart elle aussi.

65 RAMSNBES, 1949-50, 126, 135.

66 Compte rendu officiel de la Chambre des Communes, 13 Geo VI, 1949 (Ottawa, Imprimeur du Roi, 1949), III: 2548.

67 «Loi des allocations familiales», Statuts de la province de Québec. 1945, c.6, 9 Geo VI.

68 Robert Rumilly, Maurice Duplessis et son temps, tome II, 1944-1959 (Montréal, Fides), 95; Québec, Annuaire Statistique 1945-46 (Québec, Imprimeur du Roi, 1946), 183. 
provinciale, de l'autorité paternelle et des intérêts des familles nombreuses, aurait pu obtenir un certain succès ${ }^{69}$. Au terme d'une enquête menée en janvier 1948 auprès de trente-cinq familles de journaliers de Montréal, Thérèse Roy évalue l'ampleur de la discrimination à l'échelle des foyers:

L'allocation moyenne par enfant est de $\$ 8.00$ dans une famille de deux enfants et de $\$ 4.88$ dans une famille de neuf. Il nous est réellement difficile d'admettre qu'un enfant dans une famille de deux enfants puisse coûter presque le double de ce que coûte un autre enfant dans une famille de neuf... ${ }^{70}$

Mais les familles ne protesteront pas. Elles «veulent des allocations et non des discours sur l'autonomie», comme l'invoque en Chambre David Côté, seul député CCF à l'Assemblée législative, qui reçoit pour cette intervention l'approbation d'unions ouvrières ${ }^{71}$. On peut penser que, loin d'être les plus mécontentes, les familles nombreuses sont celles qui sentent le plus grand soulagement à l'occasion de la distribution des allocations. En effet, malgré le taux décroissant, plus la famille est grande, plus les allocations constituent une part imposante du revenu ${ }^{72}$ et la plupart des grandes familles vivent déjà dans un état précaire, comme en témoignent les précautions prises par les directeurs régionaux à propos des recouvrements des sommes payées en trop:

When there is a large family account, constatent-ils à l'occasion de leur réunion annuelle, complete suspension of allowances usually brings hardship. In those cases, recovery to be spread over six months automatically, rather than doing so only in exceptional circumstances. $^{73}$

Les mêmes difficultés économiques apparaissent quand Roy découvre avec étonnement que les dépenses aux chapitres du loyer, de l'alimentation, des vêtements et des loisirs «non seulement n'augmentent pas proportionnellement au nombre des enfants, mais vont même jusqu'à diminuer» ${ }^{74}$.

Une autre disposition provoque un tollé de dénonciations au Québec: le chèque au nom de la mère. Le fédéral instaure la pratique en croyant qu'il en résultera un meilleur usage des allocations pour les besoins spécifiques de chaque enfant et parfois, par conséquent, le maintien de l'enfant à la maison au lieu de son placement dans un foyer d'accueil ou une institution ${ }^{75}$. L'élite clérico-nationaliste juge la mesure

\footnotetext{
69 Simone Monet-Chartrand, Ma vie comme rivière, Tome II: 1939-1949 (Montréal, RemueMénage, 1982), 256-258.

70 Roy, op. cit., 39.

71 Rumilly, op. cit., 44-45.

72 Griffin, op. cit., 81.

73 Conference of Regional Directors of Family Allowances, May 17-20 1948, Minutes of Conference, APC, RG 29, 1934, R233/100-6125.

74 Roy, op. cit., 47.

75 RASBEBRQ, 1951-52, 12, APC, RG 29, 1283, R266/5/51.
} 
d'une tout autre façon. Pour elle, l'État a «l'intention... de transformer l'ordre social existant et de détruire l'institution familiale en s'attaquant à l'autorité paternelle» ${ }^{76}$. Duplessis s'appuie sur le code civil pour montrer que les intentions du fédéral enfreignent les règles de la communauté de bien où le père est le seul administrateur. Les pressions sont assez fortes, dans un premier temps, pour que le fédéral accepte qu'un accroc soit fait au règlement dans le cas du Québec, et le bureau régional doit reprendre 70000 dossiers déjà préparés au nom de la mère. Dans un second temps, des pressions de l'opinion publique, notamment la campagne de Thérèse Casgrain, obligent le bureau central à réviser sa décision en faveur de la mère et le bureau régional doit revoir.. 370 000 dossiers ouverts au nom du père! Ces démêlés administratifs retardent de quelques semaines l'envoi des chèques au Québec ${ }^{77}$. Par la suite, on s'attachera fermement au principe: en 1952, le tribunal d'appel des allocations refusera la demande d'un père de recevoir le chèque en son nom, malgré le consentement de l'épouse joint à la requête ${ }^{78}$. La pratique est tellement nouvelle qu'un grand nombre de chèques sont retournés par les bureaux de poste ruraux qui ne connaissent pas les prénoms des mères ${ }^{79}$.

La conception de l'autorité paternelle de l'élite conservatrice est cependant loin d'être partagée par tous les Québécois. L'Union catholique des cultivateurs et la Fédération des travailleurs du Québec prônent le paiement aux mères ${ }^{80}$. De plus, la revue Relations elle-même avait proposé, deux ans plus tôt, en éditorial, que la mère soit allocataire «pour prévenir un détournement toujours possible et pour faciliter l'application aux bénéficiaires des sommes versées tout en maintenant l'autonomie essentielle de la famille...» Elle y voyait «un hommage de confiance à l'égard de la mère du Québec» ${ }^{81}$. Gérard Parizeau, homme d'affaires et directeur de la revue Assurances, avait écrit lui aussi qu' «[u]ne bonne précaution à prendre, ce serait de verser la somme à la mère et non au père, afin d'éviter que celui-ci ne la boive» ${ }^{82}$.

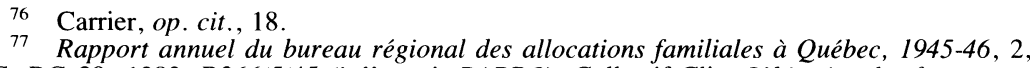
APC, RG 29, 1283, R266/5/45 (à l'avenir RABRQ); Collectif Clio, L'histoire des femmes au Québec depuis quatre siècles (Montréal, Quinze, 1982), 373-376; Monet-Chartrand, op. cit., 256258; Thérèse F. Casgrain, Une femme chez les hommes (Montréal, Éditions de l'Homme, 1971), $170-174$

${ }_{78}$ Délibérations du tribunal d'appel de Québec, séance du 22 octobre 1952, APC, RG 29, Acc. 82-83/152, 25, 3430-2-3, pt. 1. Entre sa mise sur pied, en 1948, et 1955 le tribunal reçoit six plaintes. Cette instance de recours est prévue par l'article 6 de la «Loi de 1944 sur les allocations familiales».

79 RABRQ, 1945-46, op. cit., 4.

80 Collectif Clio, op cit., 376.

81 «Le bill no 45», Relations, 3,31 (juillet 1943): 170.

82 Gérard Parizeau, «Faits d'actualité. Les allocations familiales», Assurances, 12,2 (juillet 1944): 86. On retrouve, dans un tel commentaire, un manque de confiance certain à l'égard des chefs de familles pauvres qui rappelle celui des opposants à la loi. 
Plus généralement, le mode de versement finalement retenu correspond au mode de gestion de l'économie familiale découvert par les enquêtes sur les effets des allocations familiales. Dans les trois-quarts des familles de Québec où la mère est présente, rencontrées par l'équipe de l'Université Laval, la mère seule administre le budget; dans un septième des cas, la gestion se fait en collaboration. L'équipe de McGill trouve pour sa part que plus de la moitié des mères du sud-ouest de Montréal décident seules des dépenses pour la famille et qu'un autre tiers d'entre elles décident avec leur conjoint, tandis que dans $70 \%$ des familles ouvrières montréalaises étudiées par Roy, la femme reçoit déjà le chèque de paye ${ }^{83}$. Enfin, l'équipe de McGill remarque que plus le revenu familial est élevé, plus le père exerce un contrôle sur les dépenses, ce qui explique vraisemblablement l'écart entre la conception de l'autorité paternelle de l'élite et celle de la majorité.

Il est intéressant de constater, avec Tremblay, que la plupart des enfants, sans participer aux décisions relatives aux dépenses des allocations, sont pourtant au courant de leur existence. De plus, cette conscience augmente au sein des familles à faible revenu. Certains enfants reçoivent même une partie de la prime en argent de poche et d'autres reçoivent une plus grande responsabilité au moment des emplettes: «C'est ton argent, choisis toi-même», disent certaines mères ${ }^{84}$.

En résumé, si la loi des allocations familiales peut paraître défavoriser le Québec culturellement et économiquement du fait que jusqu'en 1949 elle est basée sur la structure démographique du Canada anglais, ces désavantages sont trop faiblement ressentis à l'échelle des familles pour que naisse une protestation populaire qui aurait pu donner du poids aux démarches autonomistes de Maurice Duplessis.

\section{CONCLUSION}

Lors de la mise en oeuvre du programme des allocations familiales, les normes des législateurs et les pratiques des familles convergent plus souvent qu'elles ne s'opposent. Malgré la discrimination faite à l'endroit des autochtones et des immigrants et l'idéal bourgeois d'une famille à salaire unique qui sous-tendent la loi et ses modalités d'application, la popularité du nouveau régime montre que le gouvernement faisait preuve, en l'instaurant, d'un électoralisme bien placé. Le carac-

83 Tremblay et al., op. cit., 23. La mère ne dit pas cependant dans quelle mesure elle en discute avec son époux; Blishen et al., op . cit., 54-55; Roy, op. cit., 56. Dans les autres cas le mari paye ses comptes et donne à sa femme ce dont elle a besoin.

Tremblay et al., op . cit., 24-25. 85\% des enfants de 7 à 16 ans sont au courant. Les études de Nicolet et des Prairies mentionnent l'argent de poche sans qu'il soit possible de connaître l'étendue de la pratique. Les propos des mères sont rapportés par Jeanne Grisé-Allard, rédactrice en chef des pages féminines du Bulletin des Agriculteurs et animatrice du Courrier de Jeanne à la radio, dans «Les allocations familiales. Un chèque bien employé», Relations, 8,92 (août 1948): 240 . 
tère non discrétionnaire de la distribution des primes, la régularité des versements, la nouveauté, pour la plupart des ménages, d'un ajout public à leur revenu, la promotion de la famille naturelle, la désignation de la mère comme bénéficiaire et la propagande éducative jointe aux chèques d'allocation sont autant de facteurs qui contribuent à l'acceptation de la mesure par les familles.

Elles se montrent plus réceptives au programme et moins inquiètes de ses conséquences que la bruyante majorité de l'élite clérico-nationaliste de la province. Les conceptions qu'a cette élite de la division sexuelle du travail à l'intérieur de la famille, de l'autorité paternelle et du rôle de la famille nombreuse, de même que sa position socio-économique, l'empêchent de comprendre les besoins des familles à faible revenu. Dans le cas des allocations familiales, le rempart à l'intervention de l'État est bien plus à chercher dans l'idée que l'élite se fait des familles que chez les familles elles-mêmes. 